$$
\text { DOE/OR/00033--T664 }
$$

\title{
PATTERN RECOGNITION USING NEURAL NETWORKS
}

\author{
Prepared \\ $\mathrm{B} y$ \\ Hede Ma, Ph.D. \\ Assistant Professor \\ Department of Engineering Technology \\ Savannah State College \\ Savannah, GA 31404 \\ Phone: (912) 356-2154 \\ E-mail: hede\%uscn.bitnet@uga.cc.uga.edu \\ PECEIVED \\ JAA 231995 \\ OSTI
}

\section{Submitted}

To

\section{Ms. M. Elizabeth Kittrell}

Program Manager

Science/Engineering Education Division Oak Ridge Institute for Science and Education

$$
\text { P.O.Box } 117
$$

Oak Ridge, TN 37831-0117

\section{For}

An Appointment

in the U.S. Nuclear Regulatory Commission's

Historically' Black College and Universities

Faculty Research Participation Program

Summer 1994

(August 1 - September 11, 1994) 


\section{Contents}

Executive Summary 3

I. Introduction 4

II. Pattern Recognition 6

2.1 Dynamical Systems 7

2.2 A Pattern Recognition Scheme 7

III. Neural Networlss _9

IV Neural Networks and Pattern Recognition 11

V. Pattern Recognition Using Boltzmann Machine 12

5.1 Introduction 12

5.2 A Design of Bolt\%mann Machine for Pattern Recognition 13

5.3 An Algorithm 15

5.4 Boltzmann Machine Learning 19

5.5 Summary . 20

VI. Follow-on Research Work 21

VII. Conclusion 22

References 23

\section{DISCLAIMER}

This report was prepared as an account of work sponsored by an agency of the United States Government. Neither the United States Government nor any agency thereof, nor any of their employees, makes any warranty, express or implied, or assumes any legal liability or responsibility for the accuracy, completeness, or usefulness of any information, apparatus, product, or process disclosed, or represents that its use would not infringe privately owned rights. Reference herein to any specific commercial product, process, or service by trade name, trademark, manufacturer, or otherwise does not necessarily constitute or imply its endorsement, recommendation, or favoring by the United States Government or any agency thereof. The views and opinions of authors expressed herein do not necessarily state or reflect those of the United States Government or any agency thereof. 


\section{Executive Summary}

I am pleased to submit the following technical report to Oak Ridge National Laboratories as an accomplishment of the 6 (six) week appointment in the U.S. Nuclear Regulatory Commission's Historically Black College and Universities Faculty Research Participation Program, Summer 1994 (August 1 - September 11, 1994).

In this project, an approach for pattern recognition using neural networks is proposed. Particularly, a Boltzmann machine, a Hopfield neural net model, is used in pattern recognition with desirable learning ability. The Boltzmann machine features stochastic learning, which acts as the connection dynamics for determining the weights on the connections between the neuron-like cells (processing elements) of different layers in the neural network. An algorithm for pattern recognition using Boltzmann machine is also presented, which could be coded with $C$ programming language or others to implement the approach for efficient pattern recognition.

- Finally, a follow-on research work derived from this project is planned if the author could win another summer appointment in 1995 from the Science/Engineering Education Division, Oak Ridge Institute for Science and Education, Oak Ridge National Laboratories. 


\section{Introduction}

Neural networks with learned responses[1] can be applied to pattern recognition. Neural network models of the Hopfield type[2] have drawn intensive attention in the past years. However, the number of arbitrary vectors that can be stored in a Hopfield neural network with $N$ neurons has beeen proved to be upper bounded by $O(N)$ [3]. In order to extend this limit, the dynamical systems [4] have been classified into eight classes and introduced a pattern recognition algorithm using the third and fifth classes dynamical systems.

In this project, we will describe our approaches for pattern recognition using neural networks. A dynamical system will be used to process information. In particular, we will use the idear of processing information using stable configurations of dynamical systems developed by Hopfield in neural network theory.

The principle of simulated annealing improves the state dynamics of a Hopfield network, enabling local minima to be avoided [1]. Sinulated annealing therefore allows us to improve the 'dual' use of Hopfield networks, i.e. finding the state which gives the minimum energy of the network. For this application, it is necessary to design a connection dynamics for the initial use of such a network; a learning algorithm which allows minima to be created where desired, that is, in locations determined by the data to be memorised.

In 1985, Ackley, Hinton, and Sejnowski [5] proposed a learning algorithm for a network using simulated annealing: the Boltzmann machine.

In this project, an approach for pattern recognition using neural network is proposed. Particularly, a Boltzmann machine, a Hopfield neural net model, is used in pattern recognition with desirable learning ability. The Boltzmann machine features stochastic learning, which acts as connection dynamics for determining the weights on the connections between the neuron-like cells (processing elements) of different layers in the neural network. 
In this approach, the data encoding is to store an image in a stable distribution of a Boltzmann machine (BM). Given an input pattern $f \in F$, one can find a $B M t \in T$ such that the equilibrium distribution of this $B M$ is the given pattern $f$. Therefore, the input pattern, $f$, is encoded into a specification of a $B M, t$. This mapping from $F$ (pattern space) to $T$ (parameter space of $B M$ ) defines the BM-transformation. BM-transformation encodes an imput pattern into a relatively small vector which catches the characteristics of the input pattern. The internal space $T$ is the parameter space of $B M$. The internal change rule of our system is defined by a formula. The output data of the encoding stage is a specification of a stochastic dynamical system. Finally, an algorithm for pattern recognition using Boltzmann machine is also presented, which could be coded with $C$ programming language or others to implement the approach for efficient pattern recognition.

Section 2 introduces the basic concepts of pattern recognition. Neural networks and their applications to pattern recognition are deccribed in section 3 and section 4, respectively. Section 5 proposes an approach to pattern recognition using neural network, Boltzmann machine. Some follow-on research work are pointed out at section 6 . Finally, section 7 concludes this primary research project. 


\section{Pattern Recognition}

Pattern recognition is concerned with the classification or description by computer of objects, events, or other meaningful regularities in noisy or complex environments. Pattern recognition is the study of concepts, algorithms, and implementations that provide artificial systems with a perceptual capability to put patterns into categories in a simple and reliable way [6].

As a human experience, pattern recognition refers to a perceptual process in which patterns in any sensory modality or patterns in conceptual or logical thought processes are analyzed and recognized (or classified) as being familiar, either in the sense of having been previously experienced or of being similiar to or associated with a previous experience.

Motivation for the study of pattern recognition is (1) it is a part of the broader field of artificial intelligence, which is concerned with techniques that enable computers to do things that seem intelligent when done by people; (2) it is an important aspect of applying computers to solve problems that involve analysis and classification of measurements taken from physical process. The major applications of pattern recognition fall into three categories: (1) pattern in image (spatial pattern); (2) pattern in time (temporal pattern); and (3) pattern in more abstract data environments.

A second class dynamical system [4] is proposed as an efficient approach for pattern recognition. In the approach, the first stage is to compress a huge input vector into a tiny one that catches the characteristics of the input vector. The next stage is to process the compressed vector.

When a dynamical system has a single point attractor, it can be used as a "container" to store data. That is, given an input vector, a dynamical system can be inferred such that the attractor of the system represents the given input vector (pattern.) Furthermore, if the parameters specifying a dynamical system change continuously, so do the stored data. Therefore, if the input vectors change continuously, so do the parameters of the dynamical system. This feature can be used in real-time pattern recognition. 


\subsection{Dynamical Systems}

First of all, a dynamical system is described by a state $x \in X$ of the system. Such a state $\mathrm{x}$ is also called a configuration of the system [7]. All such configurations together form a space $H(X)$, called a configuration space. Some dynamical systems only use a subspace of $H(X)$. Secondly, an evolution of a system in its configuration space is specified by a set of production rules.

A specification of a dynamical system includes a definition of a configuration space and a set of rules for motions in the configuration space [8]. Most of dynamical system evolutions are irreversible. An orbit of a system is trajectory in its configuration space. Orbits merge with time, and after many time steps, orbits starting all possible initial configurationns become concentrated onto "attractors"[8]. These attractors typically contain only very small fraction of possible configurations. Evaluation of a dynamical system to an attractor from an arbitrary initial configuration realizes a pattern recognition.

Dynamical systems can be classified by their attractors. A class 2 dynamical system is defined as a point attractor system: there is only one configuration in the limiting case which is not a null attractor [4].

\subsection{A Pattern Recognition Scheme}

Storing data in an attractor of a dynamical system can achieve high ratio of data compression[8]. The reasons for this high ratio are (1) the attractors are relatively complex; (2) the specifications of a dynamical system are relatively simple; (3) it is possible to control a huge amount of data, specified by an attractor of a dynamical system, by manipulating a relatively small amount of data, specified by a definition of a dynamical system [9].

Given an input vecxtor or an image $x \in H(X)$, one can find a dynamical system such that the attractor of this dynamical system is the given image. Therefore, the image is compressed into a specification of a dynamical system. 
In our scheme, input vectors are processed in two stages: compression and analysis. The first stage is the data encoding: a large input vector is encoded into a relatively small vector which catches the characteristics of the input vector. The second stage only processes the encoded data which is compreesed drastically. The idea of compression is to store an input vector in an attractor of a dynamical system. Then the output data of this first stage is a specification of a dynamical system.

$\omega$-Orbit Finite Automata ( $\omega$-OFA) [9] provides a model for implementing such a pattern recognition procedure. An input vector is treated as an attractor of an $\omega$-OFA and the specification of this $\omega$-OFA is the output of the data compression stage. Once these simple rules are found, the complex input vectors can be specified by a small amount of data. Therefore, both the computation time and memory space (time complexity and space complexity) for analysis stage will be significantly reduced. This is the essence of our ideas. 


\section{Neural Networks}

Neural networks consist of three elements: (1) an organized topology (geometry) of interconnected neuron-like cells (processing elements), (2) a method of encoding information, and (3) a method of recalling information [1].

Neural network topologies, or architectures, are formed by organizing neuron-like cells into fields (also called layers) and linking them with weighted interconnections. Characteristics of these topologies include connection types, connection schemes, and field configurations. There are two primary connection types, excitatory and inhibitory. The three primary cell interconnection schemes are intra-field, interfield, and recurrent connections. Field (layer) configurations combine fields of cells, information flow and connection schemes into a coherent architecture. Field configurations include lateral feedback, field feedforward, and field feedback.

Neural network memory and learning will provide some insights concerning its encoding. Neural network memories store two types of patterns: spatial patterns and spatiotemporal patterns. There are three types of spatial pattern matching memories: random access memory (RAM), content-addressable memory (CAM), and associative memory. Neural networks act as content-addressable memory (which maps data to addresses) and associative memory (which maps data to data.) When a neural network is acting as a CAM, it stores data at stable states in some memory matrix. When a neural network is acting as an associative memory, it provides output responses from input stimuli. There are two neural network mapping mechanisms: autoassociative and heteroassociative mappings. A neural network is autoassociative if its memory stores the vectors (patterns) $X_{1}, \ldots, X_{m}$. A neural network is heteroassociative if its memory stores pattern pairs $\left(X_{1}, Y_{1}\right), \ldots,\left(X_{m}, Y_{m}\right)$.

Learning is defined to be in any change in the memory $\mathrm{W}$, i.e.

$$
\text { Learning } \equiv \frac{\mathrm{dW}}{\mathrm{dt}} \neq 0
$$


All learning methods çan be classified into (1) supervised learning, and (2) unsupervised learning. Supervised learning is a process that incorporates an external teacher and/or global information. Examples of supervised learning algorithms are errorcorrection learning, reinforcement learning, stochastic learning, and hardwired systems. Unsupervised learning, also referred to as self-organization, is a process that incorporates no external teacher and relies upon only local information and internal control.

There are two primary neural network recall mechanisms: nearest-neighbor recall and interpolative recall. Nearest-neighbor recall finds the stored input that most closely matches the stimulus and responds with the corresponding output. Interpolative recall accepts a stimulus and interpolates from the entire set of stored inputs to produce the corresponding output. 


\section{Neural Networks and Pattern Recognition}

This project discusses the application of neural networks in pattern recognition. Researchers have reported various neural network models capable of pattern recognition, models that have the function of self-organization and can learn to recognize patterns $[4$, 10]. Many are hierarchical networks consisting of layers of neuron-like cells. The ability to process information increases in proportion to the number of layers in the network.

Layered neural networks involve a set of input cells connected to a collection of output cells by means of one or more layers of modifiable intermediate connections. The most natural use of this architecture is to implement associativity by associating an input pattern, the representation of a concept or a situation, with another item, either of the same kind or totally different. For this reason, networks with multiple layers are described as associative networks.

Pattern recognition is the primary use of this mechanism. Pattern recognition is a popular application in that it enables the full set of human perceptions to be acquired by a machine.

Classically, pattern recognition is divided into a number of different stages whose relative importance varies depending on the sense being simulated and the method in use. In the context of vision, the different stages are: acquisition, concern with digitising the data coming from a sensor such as a camera, scanner or a radar detector, localising, which involves extracting the object from its background, and representation which is finding a set of significant features on the object.

The object is then represented by a real number, a word within a grammar, a graph or any element from a set of representations. The final stage, the decision stage, consists of dividing the set of object representations into number of classes.

The last two phases, representation and decision, are the associative phases. Layered neural networks can play a part in each of these phases. 


\section{Pattern Recognition Using Boltzmann Machine}

\subsection{Introduction}

Intelligent systems can learn[11]. They learn new stimuli-response dependencies. They sample the flux of experience and encode new information. They compress a huge amount of input data into small but representative internal data. In this project, we study pattern recognition using Boltzmann machines.

An approach for pattern recognition using neural network is proposed. In particular, a Boltzmann machine, a Hopfield neural net model, is used in pattern recognition with desirable learning ability. The Boltzmann machine features stochastic learning, which acts as connection dynamics for determining the weights on the connections between the neuron-like cells (processing elements) of different layers in the neural network. An algorithm for pattern recognition using Boltzmann machine is also presented, which could be coded with $C$ programming language or others to implement the approach for efficient pattern recognition.

In this approach, the data encoding is to store an image in a stable distribution of a Boltzmann machine (BM). Given an input pattern $f \in F$, one can find a $B M t \in T$ such that the equilibrium distribution of this $B M$ is the given pattern $\mathrm{f}$. Therefore, the input pattern, $f$, is encoded into a specification of a $B M$, t. This mapping from $F$ (pattern space) to $\mathrm{T}$ (parameter space of $\mathrm{BM}$ ) defines the BM-transformation. BM-transformation encodes an imput pattern into a relatively small vector which catches the characteristics of the input pattern. The internal space $T$ is the parameter space of $B M$. The internal change rule of our system is defined by a formula. The output data of the encoding stage is a specification of "a stochastic dynamical system.

Section 5.2 presents a design of Boltzmann machine for pattern recognition. An lgorithm for pattern recognition using $B M$ is shown in section 5.3. Section 5.4 discusses the learning process of BM in pattern recognition. Finally, a summary is drawn in section 5.5. 


\subsection{A Design of Boltzmann Machine for Pattern Recognition}

The Boltzmann machine $(B M)$ is a heteroassociative, nearest-neighbor pattern matcher [13] that stores arbitrary spatial patterns $\left(A_{k}, C_{k}\right), k=1,2, \ldots, m$, using a combination of Hebbian encoding and simulated annealing (i.e. stochastic learning), where the kth pattern pair is represented by the vectors $A_{k}=\left(a_{1}^{k}, \ldots, a_{n}^{k}\right)$ and $C_{k}=\left(c_{1}^{k}, \ldots, c_{q}^{k}\right)$.

The BM learns offline, operates in discrete time, and is represented by the threelayer topology shown in Fig. 1, where the $n F_{A}$ cells correspond to $A_{k}$ 's components, and the $q F_{C}$ cells correspond to $C_{k}$ 's components. The $F_{A}$ to $F_{B}$ inter-layer connections are represented by $v_{\mathrm{hi}}$, and all the $F_{B}$ to $F_{C}$ inter-layer connections are indicated with $w_{i j}$.

\section{Pattern Recognition}

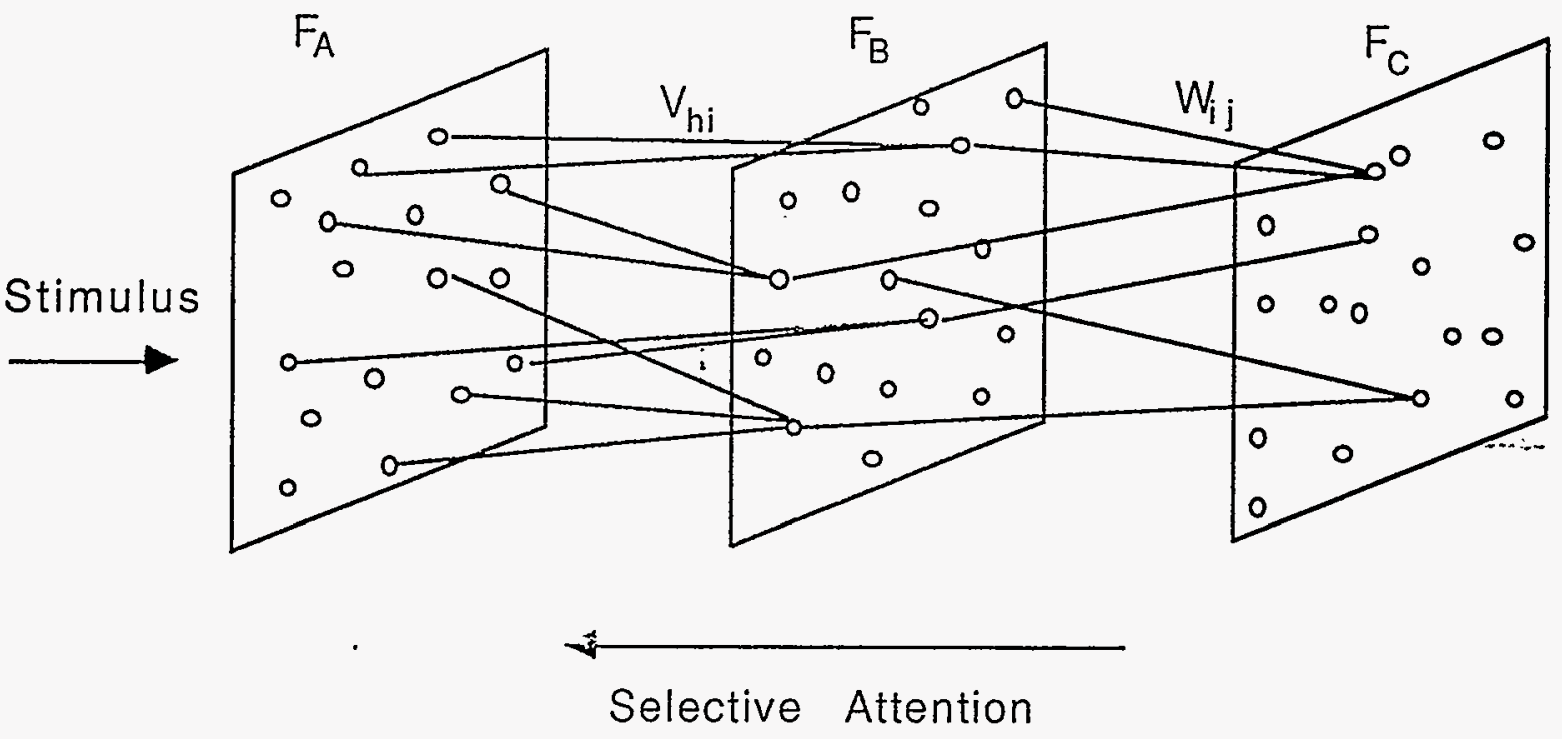

Fig. 1 A Three-Layer BM for Pattern Recognition 
Let $F$ be the set of all input patterns, $f$, e.g. $f=A_{k}$. Let $T$ be the parameter space of a family of $B M$, e.g. $t \in T$, where $t=p\left(v_{h i}, w_{i j}\right)$. Therefore, given an input pattern $f \in$ $F$, one can find a $B M$ such that the equilibrium distribution of this $B M$ is the given pattern $f$. Therefore, the input pattern, $f$, is encoded into a specification of a $B M, t$. This mapping from $F$ (pattern space) to $T$ (parameter space of BM) defines the BM-transformation. BMtransformation encodes an imput pattern into a relatively small vector which catches the characteristics of the input pattern. The internal space $T$ is the parameter space of $B M$. The internal change rule of our system is defined by a formula. The output data of the encoding stage is a specification of a stochastic dynamical system.

A stimulus pattern is presented to the first stage of the forward paths, the input layer $F_{A}$, which consists of a two-dimensional array of receptor cells. The second stage of forward paths is called the hidden layer $F_{\mathrm{B}}$. The third stage of the forward paths is the recognition layer $F_{C}$. After the process of learning ends, the final result of the pattern recognition shows in the response of the cells of $F_{A}$. In other words, cells of the recognition layer $\mathrm{F}_{\mathrm{C}}$ work as gnostic cells; usually one cell is activated, corresponding to the category of the specific stimulus pattern.

Pattern recognition by the BM occurs on the basis of similarity in shape among patterns, unaffected by deformation, changes in size, and shifts in the position of the input patterns.

The output of the recognition layer can be sent back to the first stage through the backward paths to implement selective attention (segmentation.)

Cells in BM are featuring-extracting cells. Connections converging to theses cells are variable and reinforced by learning (or training). After finishing the learning, those..... cells can extract features from the input patterns. In other words, a cell is activated only when a particular feature is presented at a certain position in the input layer. The features extracted by the cells are determined by the learning process. Generally speaking, local features, such as a line at a particular orientation, are extracted in the lower stages. More global features, such as part of a training pattern, are extracted in higher stages. Finally, each cell of the recognition layer at the highest stage integrates all the information of the input pattern; each cell responds to only one specific pattern. In other words, only one cell, corresponding to the category of the input pattern, is activated. Other cells respond to the patterns of other categories. 


\subsection{Algorithm}

The BM features stochastic learning which uses random processes, probability, and an energy relationship to adjust the memory connection weights. The BM encoding procedure incorporates a hidden $\left(\mathrm{F}_{\mathrm{B}}\right)$ layer of binary valued cell activations that are probabilistically adjusted using gradually decreasing amounts of noise to escape local energy minima in favor of the global minimum. The use of noise to escape local minima is called simulated annealing and the probabilistic assignment of hidden layer $\left(F_{B}\right)$ cell activations is called stochastic learning. An algorithm for pattern recognition using BM is given below [13].

1. Assign random values in the range $[+1,-1]$ to all the $F_{A}$ to $F_{B}$ inter-layer connections, $v_{h i}$, and all the $F_{B}$ to $F_{C}$ inter-layer connections, $w_{i j}$.

2. Starting at time $t=1$, and for each pattern pair $\left(A_{k}, C_{k}\right), k=1,2, \ldots, m$, do the following:

a. Transfer $A_{k}$ 's values to the $F_{A}$ cells and transfer $C_{k}$ 's values to the $F_{C}$ cells.

b. Randomly select an $F_{B}$ cell and switch its state, i.e. $b_{i}=1$ if $b_{i}=0, b_{i}=0$ if $b_{i}=$ 1 , where $b_{i}$ is the randomly chosen $F_{B}$ cell.

c. Calculate the change in global energy with respect to the ith $F_{B}$ cell, $\Delta E_{i}$, created by the switched state

$$
\Delta E_{i}=\sum_{h=1}^{n} v_{h i j} b_{i}+\sum_{j}^{q} w_{j=1} w_{j i}
$$

d. If $\Delta E_{i}<0$, keep the change. If $\Delta E_{i}>0$, accept the change if $P_{i}>r$, where $r$ is a number randomly chosen from $\mathrm{P}_{\mathrm{i}}$, the Boltzmann distribution given $\Delta \mathrm{E}_{\mathrm{i}}$, computed using the equation

$$
P_{i}=e-(\Delta E \mathrm{E} / T(1))
$$


where $T(t)$ is the positive valued temperature at time $t$ that regulates the search granularity for the sy'stem's global minimum (larger $T$ means courser search). If $\Delta E_{i}>0$ and $P_{i}<r$, then return $b_{i}$ to its original state prior to step (b).

e. Select a new $F_{B}$ cell and repeat steps (b) - (d).

f. Increment the time $t$ by 1 and calculate a new value for the temperature $T(t)$, using the equation

$$
T(t)=\frac{T_{0}}{1+\log t}
$$

where $T_{0}$ is the initial temperature, an equation referred to as the cooling schedule.

g. Repeat steps $(b)-(f)$ until all $\Delta E_{i}=0$. At this point the system is in equilibium and the global energy minimum has been reached.

h. Save the activation values of all the $F_{B}$ cells as a vector $D_{k}$ (for later statistical analysis of the equilibrium states) using the equation

$$
d_{i}^{k}=b_{i}
$$

for all $i=1,2, \ldots, p$, where $d_{i}^{k}$ is the value of $D_{k}$ 's ith component.

3. Using the collected equilibrium states $D_{k}$, compute the symmetric probability $Q_{h i}$ of finding both the hth $F_{A}$ and ith $F_{B}$ cells in the same state with the equation

$$
\mathrm{Q}_{\mathrm{hi}}=\frac{1}{\mathrm{~m}} \sum_{\mathrm{k}=1}^{\mathrm{m}} \Phi\left(\mathrm{a}_{\mathrm{h}}^{\mathrm{k}}, \mathrm{d}_{\mathrm{i}}^{\mathrm{k}}\right)
$$

for all $h=1,2, \ldots, n$, and all $i=1,2, \ldots, p$, where the correlation function $\Phi()$ is defineed as $\Phi(x, y)=1$ if $x=y, \Phi(x, y)=0$ otherwise, where $x$ and $y$ are binary valued scalars. In a similar fashion, computer the symmetric probability $R_{i j}$ of finding both the $j$ th $F_{C}$ and ith $F_{B}$ cells in the same state

$$
R_{i j}=\frac{1}{m} \sum_{k=1}^{m} \Phi\left(c_{j}^{k}, d_{i}^{k}\right)
$$


for all $j=1,2, \ldots, q$, and all $i=1,2, \ldots, p$.

4. Starting again at time $t=1$, and for each pattern $A_{k}, k=1,2, \ldots, m$, do the following:

a. Transfer $A_{k}$ 's values to the $F_{A}$ cells.

b. Randomly select an $F_{B}$ cell and switch its state, i.e. $b_{i}=1$ if $b_{i}=0, b_{i}=0$ if $b_{i}=$ 1 , where $b_{i}$ is the randomly chosen $F_{B}$ cell.

c. Calculate the change in global energy with respect to the ith $F_{B}$ cell, $\Delta E_{i}$, created by the switched state

$$
\Delta E_{i}=\sum_{h=1}^{n} v_{h i} b_{i}
$$

d. If $\Delta E_{i}<0$, keep the change. If $\Delta E_{i}>0$, accept the change if $P_{i}>r$, where $r$ is a number randomly chosen from $P_{i}$, the Boltzmann distribution given $\Delta E_{i}$, computed using the equation

$$
P_{i}=e-(\Delta E i / T(t))
$$

where $T(t)$ is the positive valued temperature at time $t$ that regulates the search gramularity for the system's global minimum (larger $T$ means courser search). If $\Delta E_{i}>0$ and $P_{i}<r$, then return $b_{i}$ to its original state prior to step (c).

e. Repeat steps (b)-(d) once more.

f.Increment the time $t$ and calculate a new value for the temperature $T(t)$, using the equation

$$
T(t)=\frac{T_{0}}{1+\log t}
$$

where $\mathrm{T}_{0}$ is the initial temperature, an equation referrred to as the cooling schedule.

g. Repeat steps (b)-(f) until all $\Delta E_{i}=0$. At this point the system is in equilibium and the global energy minimum has been reached.

h. Save the activation values of all the $F_{B}$ cells as a vector $D_{k}$ (for later statistical analysis of the equilibrium states) using the equation 


$$
d_{i}^{k}=b_{i}
$$

for all $i=1,2, \ldots, p$, where $d_{i}^{k}$ is the value of $D_{k}$ 's ith component.

5. Using the collected equilibrium states $D_{k}$, compute the symmetric probability $Q^{\prime}$ hi of finding both the hth $F_{A}$ and $i$ th $F_{B}$ cells in the same state with the equation

$$
Q_{h i}^{\prime}=\frac{1}{m} \sum_{k=1}^{m} \Phi\left(a_{l,}^{k}, d_{i}^{k}\right)
$$

for all $h=1,2, \ldots, n$, and all $i=1,2, \ldots, p$. In a similar fashion, compute the symmetric probability $R_{i j}^{\prime}$ of finding both the $j$ th $F_{C}$ and ith $F_{B}$ cells in the same state

$$
R_{i j}^{\prime}=\frac{1}{m} \sum_{k=1}^{m} \Phi\left(c_{j}^{k}, d_{i}^{k}\right)
$$

for all $j=1,2, \ldots, q$, and all $i=1,2, \ldots, p$.

6. Adjust the the memory matrices $V$ and $W$ using the collected statistics from the equilibrium states as follows:

a. Adjust the $F_{A}$ to $F_{B}$ connections using the equation

$$
\Delta v_{h i}=\alpha\left[Q_{h i}-Q_{h i}^{\prime}\right]
$$

where $\Delta v_{h i}$ is the change in the symmetric connection strength from the hth $F_{A}$ and the ith $F_{B}$ cell, and $\alpha$ is a positive constant controlling the learning rate.

b. Adjust the $F_{B}$ to $F_{C}$ connections using the equation

$$
\Delta w_{i j}=\alpha\left[R_{i j}-R_{i j}^{\prime}\right]
$$

where $\Delta w_{i j}$ is the change in the symmetric connection strength from the ith $F_{B}$ and the jth $F_{C}$ cell. 
7. Repeat steps (2)-(6) until $\Delta v_{h i}$ and $\Delta w_{i j}$ become sufficiently small or 0 for all $h=1,2$, . $\ldots, n$, all $i=1,2, \ldots, p$, and all $j=1,2, \ldots, q$.

\subsection{Boltzmann Machine Learning}

The BM features stochatic learning that makes a random weight change, determines the resultant energy created after the change, and keeps the weight change according to the following criteria: (1) if the neural network energy is lower after the random weight change (i.e. system performed better), accept the change, (2) if the energy is not lower after the random weight change, accept the change according to a pre-chosen probability distribution, otherwise (3) reject the change.

The BM learning rules, $\Delta v_{h i}=\alpha,\left[Q_{h i}-Q_{h i}^{\prime}\right]$ and $\Delta w_{i j}=\alpha\left[R_{i j}-R_{i j}^{\prime}\right]$, minimize an entropic measure $G_{h}$ that describes the difference between the $F_{B}$ (internal) states and $F_{A}$ and $F_{C}$ (external) states during the presentation of the hth pattern pair. This entropic cost function is defined as

$$
G_{h}=\sum_{i=1}^{n} Q_{l i l} \ln \left(Q_{h i} / Q_{h i j}^{\prime}\right)+\sum_{j=1}^{p} R_{h j} \ln \left(R_{h j} / R_{h j}^{\prime}\right)
$$

In essence, the encoding procedure attempts to capture, as well as possible, the regularities in the environment (input-output pair) via a set of connection strengths that properly perform the input-output mapping.

In fact, learning encodes information. A system learns a pattern if the system encodes the pattern in its structure. The encoding defines the internal space of a learning system and the mapping from the world space to the internal space. The mapping between the internal space and the world space is the BM-transformation (a mapping from pattern space to parameter space of $B M$ ). We will choose the parameter space $T$ of $B M$ as internal space and the BM-transformation as the mapping from the world space to the internal space.

Learning usually results in internal change of a system. That is how the internal parameters of a system change. Finally, a system adjusts its internal parameters to suit the 
new data. The internal data structure of a system must be specified. The simplest approach is a quantization of a space, which shows the internal data organization of a system.

\subsection{Summary}

To construct a learning program in pattern recognition, the statistical approach is used, which is based on the simulated annealing in BM. The learning algorithm in BM was the first model to propose solutions using hidden layer in a neural network, introducing the notion of internal representation of festures of pattern recognition. 


\section{Follow-on Research Work}

We plan to continue the following research primarily by winning another summer appointment in 1995 from Oak Ridge National Laboratories (ORNL).

1. A dynamical system composed of a set of cells (processing elements), e.g. Boltzmann machine (BM), will be designed.

2. The learning algorithm for pattern recognition using $B M$ will be coded with $C$ programming language or other programming languages the ORNL prefer.

3. The BM used for pattern recognition will be simulated.

4. The neural network, i.e. BM, will be trained for pattern recognition.

5. An experiment for recognizing input patterns will be carried out.

6. The efficiency of our design will be evaluated by the comparison with other approaches for pattern recognition using neural networks in terms of computation time and memory space.

7. The feasibility of implementing this design will be discussed for real-time application.

8. At least two publications should be resulted from this research project (one ${ }^{-\cdots \cdot}$ reports the theoretical results, another reports the results of simulation and application).

9. The total cost of the implementation, including hardware cost, software cost, and manpower cost, will be budgeted.

10. A plan for "blue-print" of this design will be worked out. 


\section{Conclusion}

In this project, we have developed a BM-learning model for pattern recognition. The model is implemented with a three layer architecture composed of a number of neuronlike cells (processing elements) and an algorithm to recognize input patterns efficiently.

This project is based on a research on the current approaches for pattern recognition using neuron networks in terms of computation time and space. The advantage of our design is that arbitrary input patterns can be recognized using the learning process with the dynamics of weighted connections linking cells in the hidden layer (treated as "black box"). The disadvantage is that the calculation power necessary to implement a BM is very large since one single step in the learning process necessitates the estimation of a probability distribution.

Since this appointment in the U.S. Nuclear Regulatory Commission's Historically Black College and Universities Faculty Research Participation Program only last six weeks, which started on 8/1/94 and ended on 9/11/94, the what we have proposed in this project needs more time (aditional appointments) to do research further and finally implement the design.

Section 6 has listed a plan for follow-on research work. The author of this research project expects another appointment at the Oak Ridge National Laboratories to continue his research work in this great topic. 


\section{References}

[1] E. Davalo and P. Naim, Neural Networks, Editions Eyrolles, Paris, 1991.

[2] J. J. Hopfield, "Neural networks and physical systems with emergent collective computation abilities," Proc. Nat. Academy Sci., USA, Vol.81, 1984, pp.3088-92.

[3] R. J. McEliece, et al., "The capacity of the Hopfield associative memory," IEEE Tran. Inform. Theory, Vol. IT33, 1987, pp.461-482.

[4] Y. Liu and H. Ma, "Pattern recognition using $\omega$-orbit finite automata," K. H. Tzao, Editor, Proc. SPIE 1606, Boston, MA, Nov. 1991, pp.226-240.

[5] D. H. Ackley, G. E. Hinton, and T. J. Sejnowski, "A learning algorithm for Boltzmann machine," Cognitive Science, Vol. 9, 1985, pp.147-169.

[6] A. Ralston and E. D. Reilly, Encyclopedia of Computer Science, Van Nostrand Reinhold, 3rd Ed., 1993.

[7] S. Wolfram, "Universality and complexity in cellular automata," Physica 10D, 1984, pp.1-35.

[8] M. F. Barnsley, Fractals Everywhere, Academic Press, 1988.

[9] Y.Liu and H. Ma, " $\omega$-Orbit finite automata for data compression," Proc. Data Compression Conference'91, IEEE Computer Press, Snowbird, Utah, April 1991, pp.165-174.

[10] T. Pawlicki, D-S. Lee, J. Hull, and S. Srihari, "Neural networks and their application to handwritten digit recognition," Proc. IEEE International Conference on Neural Networks: Vol. II, San Diego, CA, 1988, pp.63-70.

[11] B. Kosko, Neural Networks and Fuzzy Systems, Prentice Hall, 1991.

[12] Y. Liu and H. Ma, "Pattern recognition using the third and the fifth classes of dynamical systems," Pro. Sixth International Symposium on Methodology for Intelligent Systems (Poster Session), Charlotte, NC., Oct. 1991, pp.83-94.

[13] P. K. Simpson, Artificial Neural Systems, Pergamon Press, 1990.

[14] S. Amary, K. Kurata, and H. Nagaoka, "Information geometry of Boltzmann machine," IEEE Trans. Neural Network, Vol. 3, No. 2, pp.260-271. 